\title{
Comparison of the Duration of Hamstring Flexibility Improvement Following Termination of Modified Dynamic Stretching, Hold-Relax, and Static Stretching
}

\author{
A-young Moon ${ }^{1}$, BHSc, PT, Hee-jin Jang ${ }^{2}$, BHSc, PT, Hyun-jeong Jang ${ }^{3}$, MSc, PT, \\ Suhn-yeop $\mathrm{Kim}^{4}, \mathrm{PhD}, \mathrm{PT}$ \\ ${ }^{1}$ Dept. of Physical Therapy, Dongguk University Medical Center \\ ${ }^{2}$ Dept. of Physical Therapy, College of Health and Sport Science, Daejeon University \\ ${ }^{3}$ Dept. of Physical Therapy, College of Natural Science, The Graduate School, Daejeon University \\ ${ }^{4}$ Dept. of Physical Therapy, College of Natural Science, Daejeon University
}

\begin{abstract}
The aim of this study was to compare the duration of hamstring flexibility improvement after 3 stretching interventions in people with limited hamstring flexibility. Twenty-two subjects (12 men, 10 women) with limited hamstring flexibility of the dominant leg received 3 stretching interventions modified dynamic stretching (MDS), hold-relax (HR), and static stretching (SS) - in a random order. All the subjects received all 3 interventions at intervals of at least 24 hours to minimize any carry-over effect. Modified dynamic stretching was applied as a closed kinetic chain exercise in the supine position by using the sling suspension system (Redcord Trainer ${ }^{\circledR}$ ). The SS and HR interventions were individually performed in the straight leg raising (SLR) position, and all 3 interventions were performed for 3 minutes. Outcome measures included passive knee extension (PKE) measurements. Five post-test measurements were recorded for all subjects at 3, 6, 9, 15, and 30 minutes after the interventions. MDS was associated with a significant increase in knee extension range of motion even at 30 minutes post-treatment. In contrast, the HR and SS stretching methods showed increased hamstring flexibility for only 6 minutes post-treatment. Improvements in the range of motion of knee extension (indicating enhancement in hamstring flexibility) with MDS were maintained longer than those with the HR and SS interventions. Therefore, MDS may be more effective than the other interventions for maintaining hamstring flexibility.
\end{abstract}

Key Words: Duration; Dynamic stretching; Hamstring flexibility; Hold-relax; Static stretching.

\section{Introduction}

Flexibility can be defined as the ability of a muscle to lengthen and allow the related joint to move through its range of motion (ROM) (Chandler et al, 1990). Appropriate flexibility is necessary to optimize human activity (Henderson et al, 1995). Lack of flexibility has been suggested to cause a decline in the ability of the muscle to deform and may limit the body from moving appropriately, potentially facilitating musculoskeletal injury (Jonhagen et al, 1994;
Liemohn, 1978).

The hamstring muscles are an example of a muscle group with a propensity to shorten (Barash et al, 1970). Hamstring stiffness is a known compensatory mechanism for controlling undue lumbar lordosis caused by particular patterns of muscle impairments, known as "pelvic cross syndrome" (Massoud Arab et al, 2011), which may contribute to low-back dysfunction (Biering-Sørensen, 1984) and lumbar spine disorders (Barash et al, 1970). Poor flexibility of the hamstring reduces the patient's functional perform-

Corresponding author: Suhn-yeop Kim kimsy@dju.kr 
ance in various daily living activities (Jonhagen et al, 1994; Liemohn, 1978). Hamstring tightness also increases the possibility of knee flexion contracture. Knee flexion contracture can severely hinder an individual's ability to perform weight-bearing exercises and may facilitate the development of osteoporosis (Henderson et al, 1995).

Achieving flexibility has been considered to be one of the integral goals in the recovery of functional activity (Nelson, 2006; Spernoga et al, 2001). Previous stretching interventions for increasing hamstring flexibility included static stretching (SS), neuromobilization, ballistic stretching, and proprioceptive neuromuscular facilitation (PNF) (Bandy et al, 1997; Bandy and Irion, 1994; Katayama et al, 2004; Nelson and Bandy, 2004; Puentedura et al, 2011). A better option for improving flexibility, according to recent studies, would be an action that is more dynamic by nature (Murphy, 1991). Stretching with dynamic contraction has also been shown to enhance activity (Yamaguchi and Ishii, 2005). It is believed that the benefits associated with dynamic stretching are related to increased metabolic processes and post-activation potentiation. Increased muscle fiber metabolism and potentiation leads to increased muscle fiber temperature. Increased muscle temperature, in turn, reduces the viscoelasticity of the muscle-tendon unit and allows for a controlled contraction (Bishop, 2003; Sale, 2002). This warmed muscle is more extendable and more accommodating to the forces positioned on the muscle, causing enhanced flexibility gains (McMillian et al, 2006; Yamaguchi and Ishii, 2005). However, to date, the benefits of SS, PNF, and modified dynamic stretching (MDS) have not been compared.

Many field and clinical tests have been used to assess hamstring flexibility, such as the straight leg raise test, sit-and-reach tests, hip joint angle test, and the knee extension angle test (Ayala et al, 2011). The passive knee extension (PKE) test is probably the most simple and reliable method for hamstring flexibility measurement. This method also offers the benefit of minimal associated pelvic movement (Fredriksen et al, 1997).

Many studies have demonstrated the acute effects of various stretching protocols, although limited study with the specific aim of comparing duration of immediate ROM changes after stretching has been reported. These studies has shown that stretching improves flexibility and one's range of motion, regardless of stretching technique, such as ballistic, dynamic, static, or proprioceptive neuromuscular facilitation (Bandy et al, 1997; Bandy and Irion, 1994; Decoster et al, 2004; Feland et al, 2001; Puentedura et al, 2011; Yamaguchi and Ishii, 2005). However, duration of flexibility and one's range of motion gains after one-time stretching has established limited study. It would be useful to know that how long stretching can maintain muscle flexibility. No study has examined the duration of maintained hamstring flexibility following termination of modified dynamic stretching, hold-relax (HR), and static stretching.

This study investigated whether limited hamstring flexibility improved after a one-time MDS session and compared the duration of increased hamstring flexibility following MDS, HR, and SS.

\section{Methods}

\section{Subjects}

Twenty-two subjects participated in this study (Table 1). All subjects had been injury-free in the knee, thigh, hip, or the lower back region for at least 6 months prior to the study, as any injuries might have affected hamstring length or their ability to

Table 1. General characteristics of subjects $(\mathrm{N}=22)$

\begin{tabular}{cc}
\hline \hline & ${\text { Mean } \pm \mathrm{SD}^{\mathrm{a}}}^{\mathrm{A}}$ \\
\hline Age $(\mathrm{yr})$ & $22.3 \pm 1.6$ \\
Height $(\mathrm{cm})$ & $170.5 \pm 8.6$ \\
Weight $(\mathrm{kg})$ & $66.2 \pm 13.6$ \\
$\mathrm{BMI}^{\mathrm{b}}\left(\mathrm{kg} / \mathrm{m}^{2}\right)$ & $22.8 \pm 8.5$ \\
\hline
\end{tabular}

${ }^{a}$ mean \pm standard deviation, body mass index. 
perform the exercises. The dominant side in all subjects exhibited hamstring tightness, defined as a loss of $30^{\circ}$ or more from full extension with the hip at $90^{\circ}$, as delineated by Bandy and Irion (Bandy and Irion, 1994; Bandy et al, 1997; Bandy et al, 1998). All subjects read the study guidelines and gave informed consent prior to participation.

\section{Procedures}

The subjects wore shorts to allow easier palpation of the greater trochanter. Hamstring flexibility, as determined by PKE ROM, was measured in their dominant leg. The dominant leg was identified by having each subject kick a small football. Subjects were positioned supine, and a towel was placed between the lumbar spine and the examination table to help maintain lumbar curvature and minimize the influence of lumbar movements on the results. The non-dominant leg was secured to the examination table by using $10^{-} \mathrm{cm}-$ wide straps placed across the middle of the thigh. Subjects stabilized flexion of the dominant hip at $90^{\circ}$ and knee flexion at $90^{\circ}$ by using an experimental frame positioned at the posterior of the knee. The frame maintained $90^{\circ}$ hip flexion throughout PKE measurements. The available range of PKE was measured using an electronic inclinometer (PowerTrack II Commander, JTECH medical, Salt lake, USA), and a hand-held dynamometer (Dualer IQ the smarter inclinometer, JTECH medical, Salt lake, USA) was applied to maintain constant re-

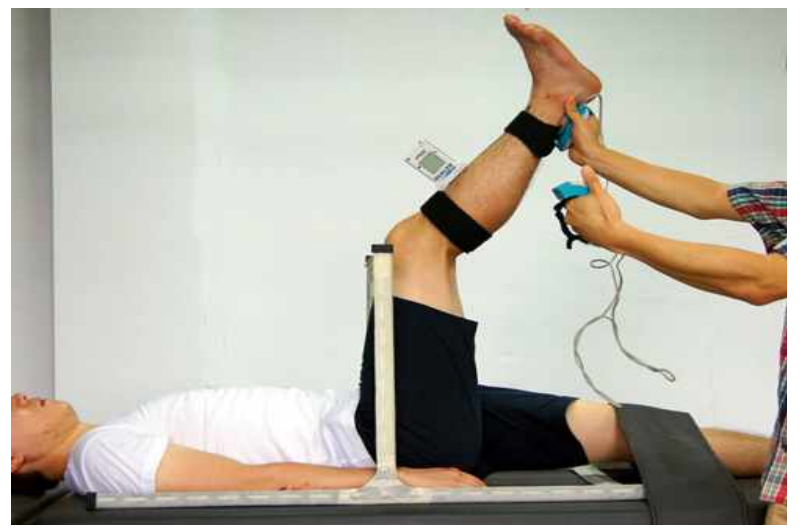

Figure 1. Measurement of hamstring flexibility. sistance (Figure 1). Electronic inclinometer and hand-held dynamometer are reliable measurements. Electronic inclinometer have intrarater reliability for intraclass correlation coefficients [ICC $(3, \mathrm{k})$ ] were $\geq .95$ (Morey, 2012), also dynamometer have reliability coefficients for test and retest measurements were .99 (Kim, 1999).

\section{Stretching methods}

The participants were asked to complete $3 \mathrm{ex}^{-}$ ercises in random order: MDS, HR, and SS. To minimize the carry-over effect between each treatment, the subjects performed each exercise after rest intervals of at least 24 hours. Each stretch intervention was performed for a total of 3 minutes (Figure 2).

\section{Modified dynamic stretching}

The MDS was executed as a closed kinetic chain exercise in a bridge position by using a sling training system (Redcord trainer, Redcord AS, Arendal, Norway). Heels were suspended by the sling training system to help the subjects maintain a bridge position with legs straight. The sling straps were maintained at ankle height with the hip and knee at $90^{\circ}$. From the straight bridge position, each subject slowly performed active knee extension and flexion exercises with a fixed anterior superior iliac spine (ASIS) height in the bridge. The subject maintained that position for an 11 seconds period with a 25 seconds rest between each repetition. The time of modified dynamic stretching intervention was applied arbitrary in order to be same with other two stretching which were applied 3 minutes totally.

\section{Hold-relax}

The HR procedure consisted of 3 stages. In the first stage, subjects lay supine with the knee fully extended. The examiner passively stretched the subject's hamstring muscles until the subject first experienced mild discomfort, and held that position for 7 seconds. In the second stage, the subject actively and isometrically contracted the muscle for 7 seconds 

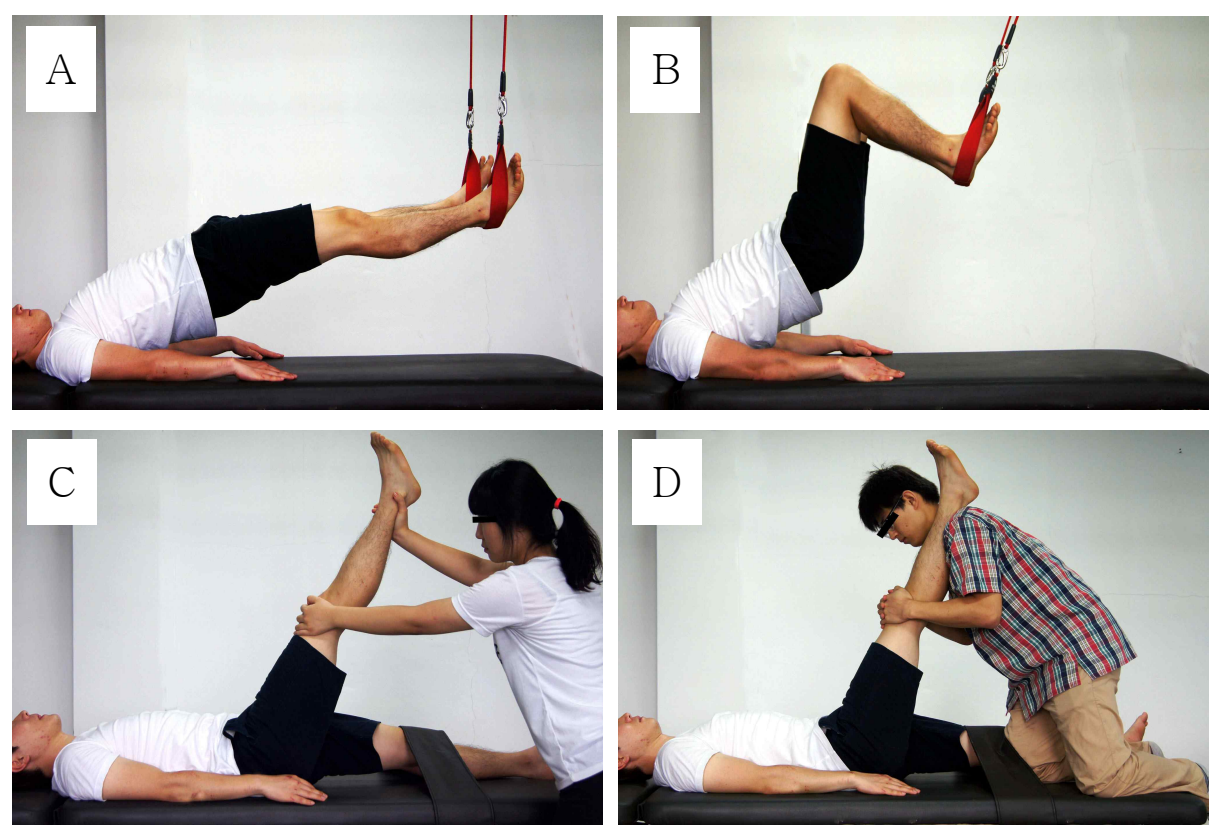

Figure 2. Three intervention interventions for improving hamstring flexibility [A: starting position of modified dynamic stretching (MDS), B: ending position of MDS, C: hold-relax, D: static stretching].

against the resistance of the investigator. In the third stage, the subject relaxed for 5 seconds. The examiner implemented an additional passive stretch to the point of discomfort for 7 seconds. For the dominant lower extremity, a set of these stretches was repeated 5 times with a 10 seconds rest interval between each trial (Boyd, 2012; Spernoga et al, 2001).

\section{Static stretching}

The subjects performed an SS-based passive SLR test. Subjects were placed supine, with their non-dominant foot planted on the floor and their knee fully extended. A researcher passively moved the dominant leg to the terminal position of knee ex$^{-}$ tension, defined as the point at which the subject complained of a feeling of discomfort. The subjects held the maximally contracted hamstring in that $\mathrm{po}^{-}$ sition for a 30 seconds period, with a 15 seconds rest interval. The test was repeated over 4 cycles (Decoster et al, 2004).

\section{Study design}

A cross-over randomized design was used. Each subject performed all 3 stretching interventions in a random order. Hamstring flexibility was measured using the PKE test. Hamstring flexibility measurements were performed pre-treatment and at 3, 6, 9, 15, and 30 minutes following treatment (Figure 3).

\section{Statistical analysis}

SPSS ver. 18.0 software (SPSS Inc., Chicago, IL, USA) was used for statistical analysis. The 3 stretching interventions within time was analyzed by one-way repeated ANOVA to determine whether

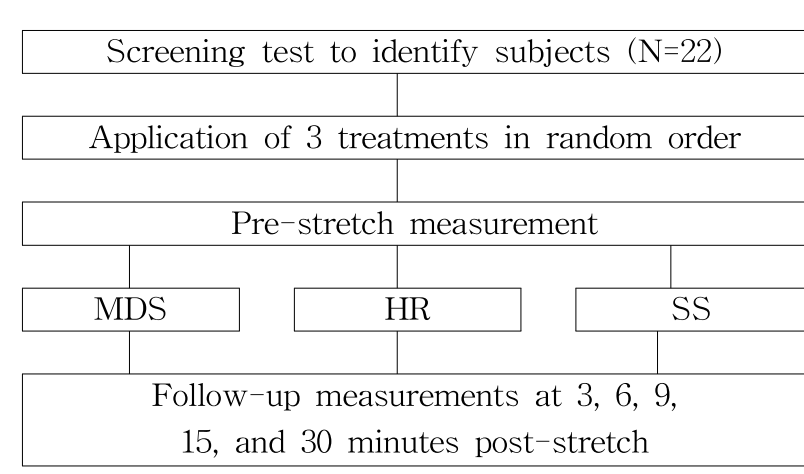

Figure 3. Flowchart illustrating the study protocol (MDS: modified dynamic stretching, HR: hold-relax, SS: static stretching). 
Table 2. Passive knee extension range of motion in degrees after MDS, HR, and SS

$(\mathrm{N}=22)$

\begin{tabular}{cccccccc}
\hline \hline $\begin{array}{c}\text { Time } \\
\text { (minute) }\end{array}$ & MDS $^{\mathrm{a}}$ & $\begin{array}{c}\text { Increment } \\
(\%)\end{array}$ & $\mathrm{HR}^{\mathrm{b}}$ & $\begin{array}{c}\text { Increment } \\
(\%)\end{array}$ & $\mathrm{SS}^{\mathrm{c}}$ & $\begin{array}{c}\text { Increment } \\
(\%)\end{array}$ & $\mathrm{F}$ \\
\hline Baseline & $58.85 \pm 6.15^{\mathrm{d}}$ & & $59.88 \pm 5.97$ & & $59.44 \pm 6.93$ & & .15 \\
Post & $64.52 \pm 6.05^{*}$ & 9.6 & $5.58 \pm 6.04^{*}$ & 9.5 & $65.88 \pm 7.57^{*}$ & 10.8 & .26 \\
3 & $64.70 \pm 6.13^{*}$ & 9.9 & $4.79 \pm 6.67^{*}$ & 8.2 & $4.14 \pm 7.52^{*}$ & 7.9 & .06 \\
6 & $65.17 \pm 6.16^{*}$ & 10.7 & $63.24 \pm 6.68^{*}$ & 5.6 & $61.92 \pm 7.57^{*}$ & 4.2 & 1.25 \\
9 & $64.58 \pm 6.21^{*}$ & 9.7 & $61.65 \pm 6.59$ & 3.0 & $60.61 \pm 7.40$ & 2.0 & 2.04 \\
15 & $64.39 \pm 6.37^{*}$ & 9.4 & $60.42 \pm 6.23$ & .9 & $59.70 \pm 7.19$ & .4 & 3.22 \\
30 & $63.24 \pm 6.59^{*}$ & 7.5 & $59.61 \pm 5.65$ & -.5 & $59.14 \pm 6.90$ & -.5 & 2.71 \\
$\mathrm{~F}$ & $2.71^{+}$ & & $3.24^{+}$ & & $2.76^{+}$ & & \\
\hline
\end{tabular}

${ }^{a}$ modified dynamic stretching, ${ }^{b}$ hold-relax, ${ }^{c}$ static stretching, ${ }^{\mathrm{d}}$ mean \pm standard deviation, ${ }^{*}$ significantly different from pre-stretch $(\mathrm{p}<.05),{ }^{\dagger}$ significantly different across groups $(\mathrm{p}<.05)$.

there were significant differences in knee-extension angle. The interaction between the 3 stretching interventions with time was analyzed by two-way repeated ANOVA. Tukey's honestly significant difference (HSD) post-hoc analysis was performed to $\mathrm{de}^{-}$ termine significant differences. The level of significance was set at $a=.05$.

\section{Results}

Our study showed that PKE ROM was significantly different after MDS, HR, and SS across the 6 time intervals $(p<.05)$. In the MDS group, a significant improvement in hamstring flexibility was maintained for 30 minutes after the stretching protocol $(\mathrm{p}<.05)$. However, the improvement in hamstring flexibility in the HR and SS groups lasted for only 6 minutes $(p<.05)$ (Table 2). Moreover, a significant intervention-by-time interaction was noted after 6 minutes by statistical analysis $(\mathrm{p}<.05)$ (Figure 4 ).

\section{Discussion}

This study was conducted to examine the duration of improved hamstring flexibility following the termination of 3 stretching interventions in subjects with limited hamstring flexibility. The results of this rela- tively small study indicate that MDS alone significantly increased hamstring flexibility for up to 30 minutes post-treatment. In contrast, while SS and HR also significantly increased hamstring flexibility, their effects reduced after just 6 minutes. The immediate effect of SS on hamstring flexibility was greater than that of the other interventions, but the difference was not statistically significant.

Previous studies reported that a 6 weeks program of dynamic stretching is helpful in increasing both

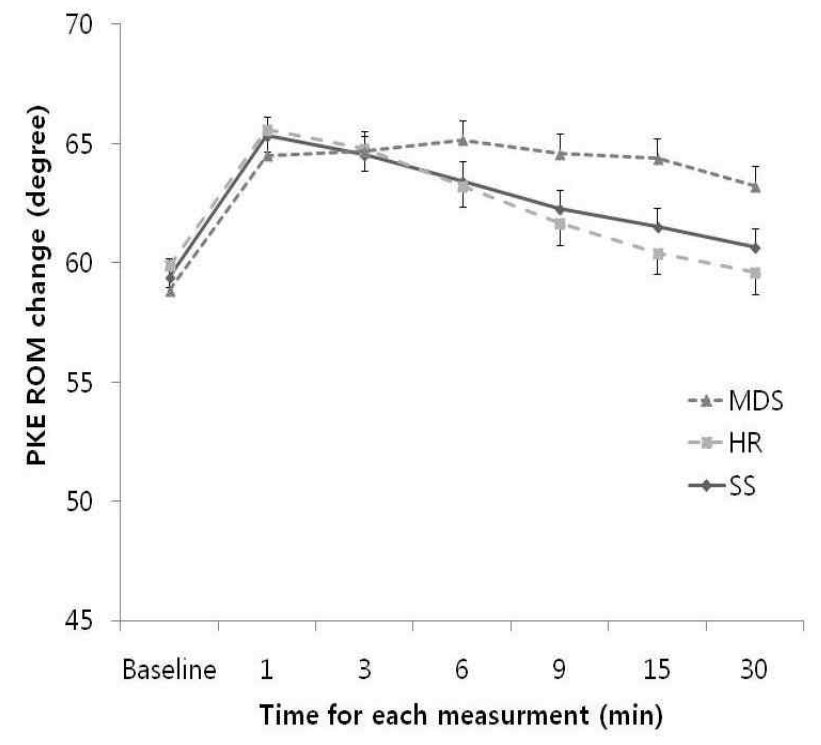

Figure 4. Passive knee extension range of motion after the 3 interventions (MDS: modified dynamic stretching, HR: hold-relax, SS: static stretching, * $\mathrm{p}>.05, T$ : standard error). 
active and passive flexibility (Nelson, 2006; O'Sullivan et al, 2009). Similarly, Nelson (2006) observed that dynamic stretching of hamstring muscles for 30 seconds (6 exercises of $1 \times 5$ seconds) was more effective than SS, and our findings corroborate this. However, Ford et al (2007) reported no difference in the duration of hamstring flexibility improvement following the termination of the 3 interventions. The discrepancy between the results of their study and our study may be explained by the following methodological differences.

Firstly, we indicated hamstring flexibility by PKE ROM values, while they used active knee extension (AKE) ROM. The reliability coefficients for test-retest measurements of the PKE and AKE tests are .99 in the right extremity and .99 for the left extremity (Fredriksen et al, 1997; Gajdosik and Lusin, 1983). Previous studies have described that hamstring flexibility values measured using AKE and PKE vary by almost $12^{\circ}$. This could be because PKE only measures "maximal hamstring length" whereas AKE measures "initial hamstring length" (Gajdosik and Lusin, 1983).

Secondly, our study applied dynamic stretching as closed kinetic chain in a bridge position, whereas the previous study used dynamic stretching as an open kinetic chain in a sitting position (Ford and McChesney, 2007). Dynamic stretching is achieved by inducing the antagonist muscle to concentrically contract in a smooth, controlled manner and thereby allow natural elongation of the agonist muscle through its full ROM (Murphy, 1991). Dynamic stretching in a closed kinetic chain improves not only flexibility but also power production. It improves post-activation potentiation, ROM, and muscle temperature (Sale, 2002). As a result, patients who receive training in dynamic contraction can achieve improvements in strength and $\mathrm{ROM}$ at the same time. Therefore, dynamic stretching as a closed kinetic chain is a more functional technique for improving flexibility (Murphy, 1991; Nelson, 2006; Snyder-Mackler, 1996). All the interventions em- ployed in our study were applied in the supine position in order to minimize the influence of other factors. Previous studies have used open kinetic chain in dynamic stretching (Ford and McChesney, 2007), whereas our research applied dynamic stretching in a bridge to create a closed kinetic chain. Lynn and Costigan (2009) reported that no difference was found in the ratio of medial-lateral hamstring muscular activation changes between hamstring bridge exercise and hamstring curl exercise. It is known that the bridge exercise is suitable for hamstring stretching.

Many studies suggested that the time and frequency of stretching protocols may have influenced the duration of the hamstring flexibility improvement (Depino et al, 2000; Feland et al, 2001; Spernoga et al, 2001). Our findings indicated that only MDS significantly improved hamstring flexibility, for up to 30 minutes, while the effects of $\mathrm{HR}$ and SS lasted for only 6 minutes. Our results differ from those obtained by Depino et al (2000), who found that the improvement in flexibility declined significantly after 3 minutes. Differences in how the SS was performed may explain this. Depino et al (2000) used a standing position during stretching, while we employed stretching in a supine position based on SLR tests. In addition, when Ford and McChesney (2007) compared the duration of hamstring flexibility improvement following the 3 interventions, they obtained results different from ours. Our subjects performed the exercises 5 times with active contraction for a period of 11 seconds and a rest period of 25 seconds, whereas the subjects in their study performed ten 10 seconds dynamic stretching exercises separated by rest periods of 10 seconds. These factors may have caused the differences between the results.

Our study was limited to the effects of MDS, HR, and SS on the flexibility of the hamstring muscles. Our sample size was small, so the results should be treated with caution and followed up in a larger study. Moreover, the group of participants observed in our study consisted of young and asymptomatic 
participants, and this finding may not be generalizable to other populations. Finally, since we measured the effect on hamstring flexibility after 3, 6, 9, 15 , and 30 minutes, the results do not elucidate any long-term effects that may persist after 30 minutes.

\section{Conclusion}

This study compared the duration of hamstring flexibility improvement after 3 different stretching interventions (MDS, HR, and SS). Twenty-two subjects (12 men, 10 women) with limited hamstring flexibility of the dominant leg received 3 stretching interventions. We found that MDS, HR, and SS were all effective in increasing the flexibility of the hamstring. However, the effects of MDS lasted up to 30 minutes after the final stretch while the effects of $\mathrm{HR}$ and SS lasted for only 6 minutes. Therefore, our finding supports the use of MDS for maintaining hamstring flexibility and provides a framework for future studies.

\section{References}

Ayala F, Sainz de Baranda P, De Ste Croix M, et al. Criterion-related validity of four clinical tests used to measure hamstring flexibility in professional futsal players. Phys Ther Sport. 2011;12(4):175-181.

Bandy WD, Irion JM. The effect of time on static stretch on the flexibility of the hamstring muscles. Phys Ther. 1994;74(9):845-852.

Bandy WD, Irion JM, Briggler M. The effect of time and frequency of static stretching on flexibility of the hamstring muscles. Phys Ther. 1997;77 (10):1090-1096.

Bandy WD, Irion JM, Briggler M. The effect of static stretch and dynamic range of motion training on the flexibility of the hamstring muscles. J Orthop Sports Phys Ther. 1998;27(4):295-300.
Barash HL, Galante JO, Lambert CN, et al. Spondylolisthesis and tight hamstrings. J Bone Joint Surg Am. 1970;52(7):1319-1328.

Biering-Sørensen F. Physical measurements as risk indicators for low-back trouble over a one-year period. Spine. 1984;9(2):106-119.

Bishop D. Warm up II: Performance changes following active warm up and how to structure the warm up. Sports Med. 2003;33(7):483-498.

Boyd BS. Measurement properties of a hand-held inclinometer during straight leg raise neurodynamic testing. Physiotherapy. 2012;98(2):174179.

Chandler TJ, Kibler WB, Uhl TL, et al. Flexibility comparisons of junior elite tennis players to other athletes. Am J Sports Med. 1990;18(2): 134-136.

Decoster LC, Scanlon RL, Horn KD, et al. Standing and supine hamstring stretching are equally effective. J Athl Train. 2004;39(4):330-334.

Depino GM, Webright WG, Arnold BL. Duration of maintained hamstring flexibility after cessation of an acute static stretching protocol. J Athl Train. 2000;35(1):56-59.

Feland JB, Myrer JW, Schulthies SS, et al. The effect of duration of stretching of the hamstring muscle group for increasing range of motion in people aged 65 years or older. Phys Ther. 2001;81(5):1110-1117.

Ford P, McChesney J. Duration of maintained hamstring rom following termination of three stretching protocols. J Sport Rehabil. 2007;16(1): 18-27.

Fredriksen H, Dagfinrud H, Jacobsen $\mathrm{V}$, et al. Passive knee extension test to measure hamstring muscle tightness. Scand J Med Sci Sports. 1997;7(5):279-282.

Gajdosik R, Lusin G. Hamstring muscle tightness. Reliability of an active-knee-extension test. Phys Ther. 1983;63(7):1085-1090.

Henderson RC, Lin PP, Greene WB. Bone-mineral density in children and adolescents who have 
spastic cerebral palsy. J Bone Joint Surg Am. 1995;77(11):1671-1681.

Jonhagen S, Nemeth G, Eriksson E. Hamstring injuries in sprinters. The role of concentric and eccentric hamstring muscle strength and flexibility. Am J Sports Med. 1994;22(2):262-266.

Katayama M, Higuchi H, Kimura M, et al. Proprioception and performance after anterior cruciate ligament rupture. Int Orthop. 2004;28(5): 278-281.

Kim JW, Lee KM. Evaluation of isometric shoulder strength in Korean adults using a hang-held dynamometer. J Korean Acad Rehabil Med. 1996;20(1):186-193.

Liemohn W. Factors related to hamstring strains. J Sports Med Phys Fitness. 1978;18(1):71-76.

Lynn SK, Costigan PA. Changes in the medial-lateral hamstring activation ratio with foot rotation during lower limb exercise. J Electromyogr Kinesiol. 2009;19(3):197-205.

Massoud Arab A, Reza Nourbakhsh M, Mohammadifar A. The relationship between hamstring length and gluteal muscle strength in individuals with sacroiliac joint dysfunction. J Man Manip Ther. 2011;19(1):5-10.

McMillian DJ, Moore JH, Hatler BS, et al. Dynamic vs. static-stretching warm up: The effect on power and agility performance. J Strenth Cond Res. 2006;20(3):492-499.

Morey JK, William JH. The reliability and concurrent validity of shoulder mobility measurements using a digital inclinometer and goniometer. Int $\mathrm{J}$ Sports Phys Ther. 2012;7(3):306-313.

Murphy DR. A critical look at static stretching: Are we doing our patient harm? Chirop Sports Med. 1991;5(3):67-70.
Nelson RT. A comparison of the immediate effects of eccentric training vs static stretch on hamstring flexibility in high school and college athletes. N Am J Sports Phys Ther. 2006;1(2):56-61.

Nelson RT, Bandy WD. Eccentric training and static stretching improve hamstring flexibility of high school males. J Athl Train. 2004;39(3):254-258.

O'Sullivan K, Murray E, Sainsbury D. The effect of warm-up, static stretching and dynamic stretching on hamstring flexibility in previously injured subjects. BMC Musculoskelet Disord. 2009;10:37.

Puentedura EJ, Huijbregts PA, Celeste S, et al. Immediate effects of quantified hamstring stretching: Hold-relax proprioceptive neuromuscular facilitation versus static stretching. Phys Ther Sport. 2011;12(3):122-126.

Sale DG. Postactivation potentiation: Role in human performance. Exerc Sport Sci Rev. 2002;30(3): 138-143.

Snyder-Mackler L. Scientific rationale and physiological basis for the use of closed kinetic chain exercise in the lower extremity. J Sport Rehabil. 1996;5(1):2-12.

Spernoga SG, Uhl TL, Arnold BL, et al. Duration of maintained hamstring flexibility after a one-time, modified hold-relax stretching protocol. J Athl Train. 2001;36(1):44-48.

Yamaguchi T, Ishii K. Effects of static stretching for 30 seconds and dynamic stretching on leg extension power. J Strenghth Cond Res. 2005;19 (3):677-683.

This article was received October 17, 2013, was reviewed October 17, 2013, and was accepted December 3, 2013. 